Pacific

Journal of

Mathematics

\title{
BRAID GROUP REPRESENTATIONS FROM TWISTED QUANTUM DOUBLES OF FINITE GROUPS
}

PAVEl ETINGOF, ERIC Rowell AND SARAH Witherspoon 


\title{
BRAID GROUP REPRESENTATIONS FROM TWISTED QUANTUM DOUBLES OF FINITE GROUPS
}

\author{
Pavel Etingof, ERic Rowell And SARAH Witherspoon
}

\begin{abstract}
We investigate the braid group representations arising from categories of representations of twisted quantum doubles of finite groups. For these categories, we show that the resulting braid group representations always factor through finite groups, in contrast to the categories associated with quantum groups at roots of unity. We also show that in the case of p-groups, the corresponding pure braid group representations factor through a finite pgroup, which answers a question asked of the first author by V. Drinfeld.
\end{abstract}

\section{Introduction}

Any braided tensor category $\mathscr{C}$ gives rise to finite dimensional representations of the braid group $\mathscr{B}_{n}$. A natural problem is to determine the image of these representations. This has been carried out to some extent for the braided tensor categories coming from quantum groups and polynomial link invariants at roots of unity [Birman and Wajnryb 1986; Franko et al. 2006; Freedman et al. 2002; Goldschmidt and Jones 1989; Jones 1986; 1989; Larsen and Rowell $\geq 2008$; Larsen et al. 2005]. A basic question in this direction is this: Is the image of the representation of $\mathscr{B}_{n}$ a finite group? In the aforementioned papers the answer is typically "no", as finite groups appear only in a few cases when the degree of the root of unity is small.

Here, we consider the braid group representations associated to the (braided tensor) categories Mod- $D^{\omega}(G)$, where $D^{\omega}(G)$ is the twisted quantum double of the finite group $G$. We show in Theorem 4.2 that the braid group images are always finite. In Theorem 4.5, we also answer in the affirmative a question of Drinfeld: If $G$ is a p-group, is the image of the pure braid group $\mathscr{P}_{n}$ also a p-group?

The content of the paper is as follows. In Section 2 we record some definitions and basic results on braided categories, and Section 3 is dedicated to the needed facts about $D^{\omega}(G)$. Then we prove our main results in Section 4. The last section describes some open problems suggested by our work.

MSC2000: primary 16W30; secondary 20F36, $18 \mathrm{D} 10$.

Keywords: twisted quantum doubles, Artin's braid group, pure braid group, representations, modular categories. 


\section{Braided categories and braid groups}

We recall some facts about braided categories and derive some basic consequences. For more complete definitions the reader is referred to either [Bakalov and Kirillov 2001] or [Turaev 1994].

The braid group $\mathscr{B}_{n}$ is defined by generators $\beta_{1}, \ldots \beta_{n-1}$ satisfying the relations

(B1) $\beta_{i} \beta_{i+1} \beta_{i}=\beta_{i+1} \beta_{i} \beta_{i+1}$ for $1 \leq i \leq n-2$, and

(B2) $\beta_{i} \beta_{j}=\beta_{j} \beta_{i}$ if $|i-j| \geq 2$.

The kernel of the surjective homomorphism from $\mathscr{B}_{n}$ to the symmetric group $S_{n}$ given by $\beta_{i} \mapsto(i, i+1)$ is the pure braid group $\mathscr{P}_{n}$, and it is generated by all conjugates of $\beta_{1}^{2}$.

Let $\mathscr{b}$ be a $k$-linear braided category over an algebraically closed field $k$ of arbitrary characteristic. The braiding structure affords us representations of $\mathscr{B}_{n}$ as follows. For any object $X$ in $\mathscr{C}$ we have braiding isomorphisms $c_{X, X} \in \operatorname{End}\left(X^{\otimes 2}\right)$, so that defining

$$
\check{R}_{i}:=\operatorname{Id}_{X}^{\otimes(i-1)} \otimes c_{X, X} \otimes \operatorname{Id}_{X}^{\otimes(n-i-1)} \in \operatorname{End}\left(X^{\otimes n}\right),
$$

we obtain a representation $\phi_{X}^{n}$ of $\mathscr{P}_{n}$ by automorphisms of $X^{\otimes n}$ by

$$
\phi_{X}^{n}\left(\beta_{i}\right)=\check{R}_{i}
$$

Similarly, for any collection of objects $\left\{X_{i}\right\}_{i=1}^{n}$, one has representations of $\mathscr{P}_{n}$ on $X_{1} \otimes \cdots \otimes X_{n}$. Throughout the paper, when we refer to representations of $\mathscr{P}_{n}$ and $\mathscr{B}_{n}$ arising from tensor products of objects in a braided category, these are the representations we mean.

We say that $Y$ is a subobject of $Z$ if there is a monomorphism $q \in \operatorname{Hom}_{\mathscr{C}}(Y, Z)$ and $W$ is a quotient object of $Z$ if there is an epimorphism $p \in \operatorname{Hom}_{\mathscr{C}}(Z, W)$. Because of the functoriality of the braiding, we have the following obvious lemma, which will be used in Section 4.

Lemma 2.1. (i) If $Y$ is a quotient object or a subobject of $Z$, then $\phi_{Y}^{n}\left(\mathscr{B}_{n}\right)$ is a quotient group of $\phi_{Z}^{n}\left(\mathscr{B}_{n}\right)$ and a similar statement holds for the restrictions of these representations to $\mathscr{P}_{n}$.

(ii) Let $S$ be a finite set of objects of a braided tensor category $\mathscr{C}$ for which the image of the representation of $\mathscr{P}_{n}$ in $\operatorname{End}\left(X_{1} \otimes \cdots \otimes X_{n}\right)$ is finite for all $X_{1}, \ldots, X_{n} \in S$. Let $X$ be the direct sum of finitely many objects taken from $S$. Then the image of the representation of $\mathscr{B}_{n}$ in $\operatorname{End}\left(X^{\otimes n}\right)$ is finite. 


\section{The twisted quantum double of a finite group}

In this section we define the twisted quantum double of a finite group and give some basic results that we need. For more details, see for example [Chari and Pressley 1994; Drinfeld 1989; Witherspoon 1996].

Let $k$ be an algebraically closed field of arbitrary characteristic $\ell$. Let $G$ be a finite group with identity element $e$, let $k G$ be the corresponding group algebra, and let $(k G)^{*}$ be the dual algebra of linear functions from $k G$ to $k$, under pointwise multiplication. There is a basis of $(k G)^{*}$ consisting of the dual functions $\delta_{g}$ for $g \in G$ defined by $\delta_{g}(h)=\delta_{g, h}(g, h \in G)$. Let $\omega: G \times G \times G \rightarrow k^{\times}$be a 3-cocycle, that is,

$$
\omega(a, b, c) \omega(a, b c, d) \omega(b, c, d)=\omega(a b, c, d) \omega(a, b, c d)
$$

for all $a, b, c, d \in G$. The twisted quantum (or Drinfeld) double $D^{\omega}(G)$ is a quasi-Hopf algebra whose underlying vector space is $(k G)^{*} \otimes k G$. We abbreviate the basis element $\delta_{x} \otimes g$ of $D^{\omega}(G)$ by $\delta_{x} \bar{g}$ for $x, g \in G$. Multiplication on $D^{\omega}(G)$ is defined by

$$
\left(\delta_{x} \bar{g}\right)\left(\delta_{y} \bar{h}\right)=\theta_{x}(g, h) \delta_{x, g y g^{-1}} \delta_{x} \overline{g h},
$$

where

$$
\theta_{x}(g, h)=\frac{\omega(x, g, h) \omega\left(g, h, h^{-1} g^{-1} x g h\right)}{\omega\left(g, g^{-1} x g, h\right)} .
$$

As an algebra, $D^{\omega}(G)$ is semisimple if and only if the characteristic $\ell$ of $k$ does not divide the order of $G$ [Witherspoon 1996].

The quasi-coassociative coproduct $\Delta: D^{\omega}(G) \rightarrow D^{\omega}(G) \otimes D^{\omega}(G)$ is defined by

$$
\Delta\left(\delta_{x} \bar{g}\right)=\sum_{\substack{y, z \in G \\ y z=x}} \gamma_{g}(y, z) \delta_{y} \bar{g} \otimes \delta_{z} \bar{g},
$$

where

$$
\gamma_{g}(y, z)=\frac{\omega(y, z, g) \omega\left(g, g^{-1} y g, g^{-1} z g\right)}{\omega\left(y, g, g^{-1} z g\right)} .
$$

The quasi-Hopf algebra $D^{\omega}(G)$ is quasitriangular with

$$
R=\sum_{g \in G} \delta_{g} \otimes \bar{g} \quad \text { and } \quad R^{-1}=\sum_{g, h \in G} \theta_{g h g^{-1}}\left(g, g^{-1}\right)^{-1} \delta_{g} \bar{e} \otimes \delta_{h} \overline{g^{-1}} .
$$

In particular $R \Delta(a) R^{-1}=\sigma(\Delta(a))$ for all $a \in D^{\omega}(G)$, where $\sigma$ is the transposition map. If $X$ and $Y$ are $D^{\omega}(G)$-modules, then $\check{R}=\sigma \circ R$ provides a $D^{\omega}(G)$-module isomorphism from $X \otimes Y$ to $Y \otimes X$. Let $c_{X, Y}$ be this action by $\check{R}$. Then the category Mod- $D^{\omega}(G)$ of finite dimensional $D^{\omega}(G)$-modules is a braided category with braiding $c$. 


\section{The images of $\mathscr{B}_{n}$ and $\mathscr{P}_{n}$}

In this section we fix a finite group $G$ and a 3-cocycle $\omega$, and we prove that the image of $\mathscr{B}_{n}$ in $\operatorname{End}_{D^{\omega}(G)}\left(V^{\otimes n}\right)$ is finite for any positive integer $n$ and any finite dimensional $D^{\omega}(G)$-module $V$. When $G$ is a $p$-group, we prove that the image of $\mathscr{P}_{n}$ in $\operatorname{End}_{D^{\omega}(G)}\left(V^{\otimes n}\right)$ is also a $p$-group.

Remark 4.1. It follows from a theorem of C. Vafa, see [Bakalov and Kirillov 2001, Theorem 3.1.19], and the so-called balancing axioms that for braided fusion categories over $\mathbb{C}$, the images of the braid group generators $\beta_{i}$ in the above representations of $\mathscr{B}_{n}$ always have finite order. This is far from enough to conclude that the image of $\mathscr{B}_{n}$ is finite; Coxeter [1959] has shown that the quotient of $\mathscr{B}_{n}$ by the normal closure of the subgroup generated by $\left\{\beta_{i}^{k}: 1 \leq i \leq n-1\right\}$ is finite if and only if $1 / n+1 / k>1 / 2$.

The case of general finite groups. Let $r$ and $m$ be positive integers. The full monomial group $G(r, 1, m)$ is the multiplicative group consisting of the $m \times m$ matrices having exactly one nonzero entry in each row and column, all of whose nonzero entries are $r$-th roots of unity. It is one of the irreducible complex reflection groups.

Let $r=|G|_{\ell^{\prime}}$ be the part of $|G|$ not divisible by the characteristic $\ell$ of $k$, that is, $|G|=r \ell^{s}$ and $(r, \ell)=1$.

Theorem 4.2. Let $V$ be a finite dimensional $D^{\omega}(G)$-module. Then the image of $\mathscr{B}_{n}$ in $\operatorname{End}\left(V^{\otimes n}\right)$ is finite. More specifically, this image is a quotient of a subgroup of $G(r, 1, m)$ for $m=|G|^{2 n}$.

Proof. We will need the following well-known lemma, which follows from [Weibel 1994, Theorem 6.5.8]. Let $\mu_{r} \subset k^{\times}$be the set of $r$-th roots of unity.

Lemma 4.3. The natural map $H^{i}\left(G, \mu_{r}\right) \rightarrow H^{i}\left(G, k^{\times}\right)$is surjective. In particular, any element in $H^{i}\left(G, k^{\times}\right)$may be represented by a cocycle taking values in $\mu_{r}$.

Now we turn to the proof of the theorem. As any finite dimensional $D^{\omega}(G)$ module is finitely generated and is therefore a quotient of a finite rank free module, by Lemma 2.1(i) it suffices to prove the statement when $V$ is a finite-rank free module. By Lemma 2.1(ii), we need only consider the case $V=D^{\omega}(G)$, the left regular module.

Assume first that $n=2$. Let $x, y, a, b \in G$. The action of $\check{R}$ on the basis element $\delta_{x} \bar{a} \otimes \delta_{y} \bar{b}$ of $D^{\omega}(G) \otimes D^{\omega}(G)$ is

$$
\begin{aligned}
\check{R}\left(\delta_{x} \bar{a} \otimes \delta_{y} \bar{b}\right)=\sigma\left(\sum_{g \in G} \delta_{g} \otimes \bar{g}\right)\left(\delta_{x} \bar{a} \otimes \delta_{y} \bar{b}\right) & =\sigma\left(\theta_{x y x^{-1}}(x, b) \delta_{x} \bar{a} \otimes \delta_{x y x^{-1}} \overline{x b}\right) \\
& =\theta_{x y x^{-1}}(x, b) \delta_{x y x^{-1}} \overline{x b} \otimes \delta_{x} \bar{a}
\end{aligned}
$$


If $n>2$, similar calculations show that each $\check{R}_{i}$ permutes the chosen basis of $D^{\omega}(G)$ up to scalar multiples of the form $\theta_{x y x^{-1}}(x, b)$. By Lemma 4.3, we may assume that $\omega$ and hence $\theta$ takes values in the $r$-th roots of unity. This implies that the image of $\mathscr{B}_{n}$ in $\operatorname{End}\left(D^{\omega}(G)^{\otimes n}\right)$ is contained in $G(r, 1, m)$.

Corollary 4.4. Let $\mathscr{C}$ be a braided fusion category that is group theoretical in the sense of [Etingof et al. 2005]. Let $V$ be any object of $\mathscr{C}$. Then the image of $\mathscr{B}_{n}$ in $\operatorname{End}\left(V^{\otimes n}\right)$ is finite.

Proof. Let $Z(\mathscr{b})$ be the Drinfeld center of $\mathscr{C}$. Since $\mathscr{C}$ is braided, we have a canonical braided tensor functor $F: \mathscr{C} \rightarrow Z(\mathscr{C})$. Thus it suffices to show the result holds for the category $Z(\mathscr{b})$. Since $\mathscr{C}$ is group theoretical, $Z(\mathscr{b})$ is equivalent to Mod - $D^{\omega}(G)$ for some $G, \omega$; see [Natale 2003]. Thus the desired result follows from Theorem 4.2.

\section{The case of p-groups.}

Theorem 4.5. Suppose that $G$ is a finite $p$-group and $V$ is a finite dimensional $D^{\omega}(G)$-module. Then the image of $\mathscr{P}_{n}$ in $\operatorname{End}\left(V^{\otimes n}\right)$ is also a p-group.

The rest of the subsection is occupied by the proof of Theorem 4.5. We will need a technical lemma:

Lemma 4.6. Let $H$ be a group with normal subgroups $H=H_{0} \supset H_{1} \supset \ldots \supset H_{N}=1$ such that $H_{i} / H_{i+1}$ is abelian and $\left[H_{i}, H_{j}\right] \subset H_{i+j}$, and let $I$ be a subgroup of $\operatorname{Aut}(H)$ that preserves this filtration and acts trivially on the associated graded group. Then I is nilpotent of class at most $N-1$.

Proof. Let $L_{1}(I)=I, L_{2}(I)=[I, I], L_{3}(I)=[[I, I], I], \ldots$ be the lower central series of $I$. We must show $L_{N}(I)=1$.

We prove by induction on $n$ that for any $f \in L_{n}(I)$ and $h \in H_{m}$, we have $f(h)=h a(h)$, where $a(h) \in H_{n+m}$.

The case $n=1$ is clear, since $f \in I$ acts trivially on $H_{m} / H_{m+1}$. Suppose the statement is true for $n$. Take $g \in I, f \in L_{n}(I)$, and $h \in H_{m}$ such that $f(h)=$ $h a(h)$ and $g(h)=h b(h)$, where $a(h) \in H_{n+m}$ and $b(h) \in H_{m+1}$. Then $f g(h)=$ $f(h) f(b(h))=h a(h) b(h) a(b(h))$, while $g f(h)=h b(h) a(h) b(a(h))$.

Since $g$ acts trivially on the associated graded group, we have $b(a(h)) \in H_{n+m+1}$. Also $a(b(h)) \in H_{n+m+1}$ since $b(h) \in H_{m+1}$, by the induction assumption. Also $a(h) b(h)=b(h) a(h)$ modulo $H_{n+m+1}$ since $\left[H_{i}, H_{j}\right] \subset H_{i+j}$. Thus $f g(h)=g f(h)$ in $H / H_{n+m+1}$, and thus $[f, g](h)=h$ in $H / H_{n+m+1}$, which is what we needed to show.

Taking $m=0$ and $n=N-1$, any $[f, g] \in L_{N}(I)$ is the identity on $H=H / H_{N}$, and the lemma is proved. 
Proof of Theorem 4.5. Any finite dimensional $D^{\omega}(G)$-module is a quotient of a multiple of the left regular $D^{\omega}(G)$-module $H=D^{\omega}(G)$. By Lemma 2.1, it suffices to show that the image of $\mathscr{P}_{n}$ in $\operatorname{End}\left(H^{\otimes n}\right)$ is a $p$-group. By Theorem 4.2, the image $K$ of $\mathscr{P}_{n}$ is a subgroup of the full monomial group $G(r, 1, m)$, where $r=p^{t}$ for some $t$, and $m=|G|^{2 n}$. The normal subgroup of diagonal matrices in $K$ is thus a $p$-group, and so it is enough to show that $K$ modulo the diagonal matrices is a $p$-group. Thus it suffices to assume that $\omega=1$ and $H=D(G)$.

Computing, we have

$$
\check{R}\left(\bar{a} \delta_{x} \otimes \bar{b} \delta_{y}\right)=\sigma\left(\sum_{g \in G} \delta_{g} \otimes \bar{g}\right)\left(\bar{a} \delta_{x} \otimes \bar{b} \delta_{y}\right)=\overline{a x a^{-1} b} \delta_{y} \otimes \bar{a} \delta_{x}
$$

for all $a, b, x, y \in G$. Denote by $(g, x)$ the element $\bar{g} \delta_{x}$ so that a basis of $H^{\otimes n}$ is

$$
\left(g_{1}, x_{1}\right) \otimes \cdots \otimes\left(g_{n}, x_{n}\right)
$$

with $g_{i}, x_{i} \in G$. The braid generator $\beta_{i}$ fixes all factors other than the $i$-th and $(i+1)$-st, and on these it acts by

$$
\begin{aligned}
\left(g_{i}, x_{i}\right) \otimes\left(g_{i+1}, x_{i+1}\right) & \mapsto\left(g_{i} x_{i} g_{i}^{-1} x_{i+1}, x_{i+1}\right) \otimes\left(g_{i}, x_{i}\right) \\
& =\left(\left[g_{i}, x_{i}\right] x_{i} g_{i+1}, x_{i+1}\right) \otimes\left(g_{i}, x_{i}\right),
\end{aligned}
$$

where $[a, b]$ denotes the group commutator. This action induces a homomorphism $\psi: \mathscr{B}_{n} \rightarrow \operatorname{Aut}\left(\operatorname{Fr}_{2 n}\right)$ where $\mathrm{Fr}_{2 n}$ is the free group on $2 n$ generators. Explicitly, $\psi\left(\beta_{i}\right)$ is the automorphism defined on generators $\left\{g_{i}, x_{i}\right\}_{i=1}^{n}$ of $\mathrm{Fr}_{2 n}$ by

$$
\begin{aligned}
x_{j} & \mapsto x_{j}, \quad g_{j} \mapsto g_{j} \quad \text { for } j \notin\{i, i+1\}, \\
x_{i} & \mapsto x_{i+1}, \quad x_{i+1} \mapsto x_{i}, \quad g_{i} \mapsto\left[g_{i}, x_{i}\right] x_{i} g_{i+1}, \quad g_{i+1} \mapsto g_{i} .
\end{aligned}
$$

Since $G$ is a $p$-group, it is nilpotent of class, say, $N-1$. Note that $\psi$ descends to a homomorphism $\psi_{N}: \mathscr{B}_{n} \rightarrow \operatorname{Aut}\left(\operatorname{Fr}_{2 n} / L_{N}\left(\operatorname{Fr}_{2 n}\right)\right)$ where $L_{N}\left(\operatorname{Fr}_{2 n}\right)$ denotes the $N$-th term of the lower central series of $\operatorname{Fr}_{2 n}$. Since $G$ is nilpotent of class $N-1$, the action of $\mathscr{B}_{n}$ on the set $G^{2 n}$ defined above factors through $\psi_{N}$. Thus, setting $I=\psi_{N}\left(\mathscr{P}_{n}\right)$, one sees that the action of $\mathscr{P}_{n}$ on $H^{\otimes n}$ factors through $I$, that is, $K$ is a quotient of $I$.

Let us now show that $I$ is nilpotent. Define a descending filtration on $M=$ $\mathrm{Fr}_{2 n} / L_{N}\left(\mathrm{Fr}_{2 n}\right)$ by positive integers as follows. Let $M_{1}=M$. Define degrees on the generators by $\operatorname{deg}\left(g_{i}\right)=1$ and $\operatorname{deg}\left(x_{i}\right)=2$ for all $i$, and define $M_{j}$ for $j \geq 2$ to be the normal closure of the group generated by $\left[M_{k}, M_{j-k}\right]$ for all $0 \leq k \leq j$ together with the generators of degree at least $j$. Since $M$ is nilpotent, this filtration is finite. Further, $I$ preserves this filtration and acts trivially on the quotients $M_{i} / M_{i+1}$. By Lemma 4.6, $I$ is nilpotent. 
It follows that the finite group $K$ is nilpotent. However, $K$ is generated by conjugates of $\beta_{1}^{2}$, and we claim that $\beta_{1}^{2}$ is an element whose order is a power of $p$. Indeed, this follows from the fact that if the ground field is $\mathbb{C}$ (which may be assumed without loss of generality, since the double of $G$ is defined over the integers), then the eigenvalues of $c_{X, Y} c_{Y, X}$ for any objects $X$ and $Y$ are ratios of twists, which are computed from characters of $G$ (in [Bakalov and Kirillov 2001]), and hence are roots of unity whose degrees are powers of $p$. Therefore, $K$ is a finite $p$-group.

\section{Questions}

We mention some directions for further investigation suggested by these (and other) results. We refer the reader to [Etingof et al. 2005] and [Turaev 1994] for the relevant definitions.

(1) Suppose $G$ is a $p$-group. Theorem 4.5 shows that the image of the associated representation of $\mathscr{P}_{n}$ is also a $p$-group. What is its nilpotency class relative to that of $G$ ? Some upper bounds can be obtained from the proof of Theorem 4.5 , but it is not clear how tight they are.

(2) The finite groups that appear as images of representations of $\mathscr{B}_{n}$ associated to quantum groups and link invariants at roots of unity (see [Franko et al. 2006; Goldschmidt and Jones 1989; Jones 1986; 1989; Larsen and Rowell $\geq 2008$; Larsen et al. 2005]) basically fall into two classes: symplectic groups and extensions of $p$-groups by the symmetric group $S_{n}$. Does this hold for the representations of $\mathscr{B}_{n}$ associated with $\operatorname{Mod}-D^{\omega}(G)$ ? In general, is there a relationship between the image of $\mathscr{P}_{n}$ and $G$ ?

(3) As a modular category, Mod - $D^{\omega}(G)$ gives rise to (projective) representations of mapping class groups of compact surfaces with boundary. Are the images always finite? It is known to be true for the mapping class groups of the torus and the $n$-punctured sphere (Theorem 4.2). For more general modular categories, the answer is definitely "no." See [Andersen et al. 2006, Conjecture 2.4].

(4) Let us say that a braided category $\mathscr{C}$ has property $\mathscr{F}$ if all braid group representations associated to $\mathscr{C}$ have finite images. What class of braided categories have property $\mathscr{F}$ ? Among braided fusion categories, Corollary 4.4 shows that all braided group-theoretical categories, in the sense of [Etingof et al. 2005], have property $\mathscr{F}$. Do all braided fusion categories with integer FrobeniusPerron dimension have property $\mathscr{F}$ ? 


\section{Acknowledgments}

Etingof is grateful to V. Drinfeld for a useful discussion and for raising the question answered by Theorem 4.5. His work was partially supported by NSF grant DMS0504847. Witherspoon was partially supported by NSA grant H98230-07-1-0038.

\section{References}

[Andersen et al. 2006] J. E. Andersen, G. Masbaum, and K. Ueno, "Topological quantum field theory and the Nielsen-Thurston classification of M(0,4)", Math. Proc. Cambridge Philos. Soc. 141:3 (2006), 477-488. MR 2007k:57059 Zbl 1110.57009

[Bakalov and Kirillov 2001] B. Bakalov and A. Kirillov, Jr., Lectures on tensor categories and modular functors, University Lecture Series 21, American Mathematical Society, Providence, RI, 2001. MR 2002d:18003 Zbl 0965.18002

[Birman and Wajnryb 1986] J. S. Birman and B. Wajnryb, "Markov classes in certain finite quotients of Artin's braid group”, Israel J. Math. 56:2 (1986), 160-178. MR 88b:20063 Zbl 0621.20025

[Chari and Pressley 1994] V. Chari and A. Pressley, A guide to quantum groups, Cambridge University Press, Cambridge, 1994. MR 95j:17010 Zbl 0839.17009

[Coxeter 1959] H. S. M. Coxeter, "Factor groups of the braid group", pp. 95-122 in Proceedings of the Fourth Can. Math. Cong. (Banff 1957), University of Toronto Press, 1959. Zbl 0093.25003

[Drinfeld 1989] V. G. Drinfeld, “Quasi-Hopf algebras”, Algebra i Analiz 1:6 (1989), 114-148. MR 91b:17016

[Etingof et al. 2005] P. Etingof, D. Nikshych, and V. Ostrik, "On fusion categories", Ann. of Math. (2) 162:2 (2005), 581-642. MR 2006m:16051 Zbl 05042683

[Franko et al. 2006] J. M. Franko, E. C. Rowell, and Z. Wang, "Extraspecial 2-groups and images of braid group representations", J. Knot Theory Ramifications 15 (2006), 413-427. MR 2006m:20053 Zbl 1097.20034

[Freedman et al. 2002] M. H. Freedman, M. J. Larsen, and Z. Wang, "The two-eigenvalue problem and density of Jones representation of braid groups", Comm. Math. Phys. 228:1 (2002), 177-199. MR 2004d:20037 Zbl 1045.20027

[Goldschmidt and Jones 1989] D. M. Goldschmidt and V. F. R. Jones, "Metaplectic link invariants", Geom. Dedicata 31:2 (1989), 165-191. MR 91e:57014 Zbl 0678.57007

[Jones 1986] V. F. R. Jones, "Braid groups, Hecke algebras and type $\mathrm{II}_{1}$ factors", pp. 242-273 in Geometric methods in operator algebras (Kyoto, 1983), edited by H. Araki and E. G. Effros, Pitman Res. Notes Math. Ser. 123, Longman Sci. Tech., Harlow, 1986. MR 88k:46069 Zbl 0659.46054

[Jones 1989] V. F. R. Jones, "On a certain value of the Kauffman polynomial”, Comm. Math. Phys. 125:3 (1989), 459-467. MR 91e:57017 Zbl 0695.57003

[Larsen and Rowell $\geq 2008$ ] M. J. Larsen and E. C. Rowell, "An algebra-level version of a linkpolynomial identity of Lickorish", Math. Proc. Cambridge Philos. Soc.. To appear.

[Larsen et al. 2005] M. J. Larsen, E. C. Rowell, and Z. Wang, "The $N$-eigenvalue problem and two applications”, Int. Math. Res. Not. 64 (2005), 3987-4018. MR 2006m:22019 Zbl 05030168

[Natale 2003] S. Natale, "On group theoretical Hopf algebras and exact factorizations of finite groups”, J. Algebra 270:1 (2003), 199-211. MR 2004k:16102 Zbl 1040.16027

[Turaev 1994] V. G. Turaev, Quantum invariants of knots and 3-manifolds, de Gruyter Studies in Mathematics 18, Walter de Gruyter \& Co., Berlin, 1994. MR 95k:57014 Zbl 0812.57003 
[Weibel 1994] C. A. Weibel, An introduction to homological algebra, Cambridge Studies in Advanced Mathematics 38, Cambridge University Press, 1994. MR 95f:18001 Zbl 0797.18001

[Witherspoon 1996] S. J. Witherspoon, "The representation ring of the twisted quantum double of a finite group”, Canad. J. Math. 48:6 (1996), 1324-1338. MR 97m:16078 Zbl 0876.16027

Received March 9, 2007.

PAVEl ETINGOF

Department of Mathematics

Massachusetts Institute of TeChNOLOGY

77 Massachusetts Ave.

CAMBRIDGE, MA 02139-4307

etingof@math.mit.edu

\section{ERIC ROWELL}

DEPARTMENT OF MATHEMATICS

TEXAS A\&M UNIVERSITY

College Station, TX 77843-3368

rowell@math.tamu.edu

SARAH WITHERSPOON

DEPARTMENT OF MATHEMATICS

TEXAS A\&M UNIVERSITY

College Station, TX 77843-3368

sjw@math.tamu.edu 